\title{
Adolescent pregnancy and associated factors in South African youth
}

\author{
Mchunu $\mathrm{G}^{1}$, Peltzer $\mathrm{K},{ }^{1,2 *}$ Tutshana $\mathrm{B}^{1}$, Seutlwadi $\mathrm{L}^{1}$
}

1. HIV/AIDS, TB and STI (HAST) Research Programme, Human Sciences Research Council, Pretoria and Durban, South Africa

2. University of Limpopo, Turfloop, South Africa

\begin{abstract}
Background: Adolescent pregnancy, occurring in girls aged 10-19 years, remains a serious health and social problem worldwide, and has been associated with numerous risk factors evident in the young people's family, peer, school, and neighbourhood contexts.

Objective: 'To assess the prevalence of adolescent pregnancy and associated factors in the South African context, as part of a population-based household survey that formed part of an evaluation of the impact of loveLife, South Africa's national HIV prevention campaign for young people.

Methods: A cross-sectional population-based household survey was conducted using a multi-stage stratified cluster sampling approach. The total sample included 3123 participants, aged 18-24, 54.6\% men and 45.4\% women, from four of nine provinces in South Africa (Eastern Cape, Gauteng, KwaZulu-Natal and Mpumalanga).

Results: Among female youth $19.2 \%$ said that they had an adolescent pregnancy, while $5.8 \%$ of male youth indicated that they had impregnated a girl when they were an adolescent (12-19 years), 16.2\% of the women indicated that they ever had an unwanted pregnancy and $6.7 \%$ had ever terminated a pregnancy. In multivariable analysis among women it was found that being employed or unemployed, greater poverty, having higher sexually permissive attitudes and scoring higher on the contraceptive or the condom use index was associated with adolescent pregnancy, and among men wanting the pregnancy and having a sense of the future were associated with adolescent pregnancy.

Conclusion: Adolescent pregnancy was found to be high in this sample of South African youth. Multiple factors contributing to adolescent pregnancy have been identified which can be used in targeting young people on the prevention of adolescent pregnancy.
\end{abstract}

Key words: Adolescent pregnancy; youth between 18-24 years, loveLife youth programme exposure, South Africa African Health Sciences 2012; (4): 426 - 434 http://dx.doi.org/10.4314/ahs.v12i4.5

\section{Introduction}

Adolescent pregnancy, occurring in girls aged 1019 years, remains a serious health and social problem worldwide, and has been associated with numerous risk factors evident in the young people's family, peer, school, and neighbourhood contexts. ${ }^{1-3}$ In subSaharan Africa, one such risk factor in early child bearing is increased vulnerability to HIV/AIDS. While HIV is one of the unintended consequences of unprotected sexual intercourse, pregnancy is another indicator that young people are having unprotected sex.

\section{*Corresponding author: \\ Prof Karl Peltzer \\ HIV/AIDS, TB and STI (HAST) \\ Human Sciences Research Council \\ Private Bag X41, Pretoria 0001 \\ South Africa \\ Email: KPeltzer@hsrc.ac.za}

Evidence from sub-Saharan Africa indicates that $35 \%$ of pregnancies among $15-19$ year olds were unplanned, unwanted or untimed and that the teenagers' relationships were unstable. ${ }^{4,5}$ Only about two thirds of these unintended pregnancies end in childbirth, while a third results in unsafe abortions. ${ }^{5}$ A study conducted in Soweto, South Africa, found that $23 \%$ of pregnancies carried by 13-16 year old young women and $14.9 \%$ in the $17-19$ year age range ended in abortion. ${ }^{6}$ Several studies have identified the predictors of unsafe sexual practices during the early adolescent years, such as individual, sociodemographic, familial, and relational characteristics, poverty, cultural and family patterns of early sexual experience and lack of school or career goals. ${ }^{7,8}$

Adolescent pregnancy interferes with young women's educational attainment, resulting in fewer job opportunities for young women. ${ }^{9}$ Several studies have argued that young school girls engage in sex

African Health Sciences Vol 12 Issue 4 December 2012 
with older partners and have transactional sex, whereby gift or money are exchanged for sex. ${ }^{10-13}$ Such relationships result in young women having little or no negotiating power with their partners to insist on condoms usage a situation which may result in high risk of becoming pregnant and contracting sexually transmitted infections (STIs), including HIV / AIDS. ${ }^{14,15}$ Access to reproductive health services is another factor which contributes to adolescent pregnancy since young people always want to be able access sexual and reproductive health information and services without being exposed to public stigma. ${ }^{16}$ Many studies on teenage pregnancy have focused on the practices of adolescents in general and outcomes of their pregnancies, but very limited understanding of factors that place particular adolescents at increased risk of teenage pregnancy. ${ }^{17}$ Therefore, there is a need for studies that focus on factors that put adolescents at risk of teenage pregnancy from both female and male partners. Therefore, the aim of our study was to investigate the prevalence adolescent pregnancy and associated factors in the South African context, as part of a large population-based household survey that formed part of an evaluation of the impact of loveLife, South Africa's national HIV prevention campaign for young people.

\section{Method}

\section{Sample and procedure}

We conducted a cross-sectional population-based household survey using a multi-stage stratified cluster sampling approach. A total of 289 census enumeration areas (EAs) from the 2001 population census were selected from a database of 86000 EAs and mapped in 2007 using aerial photography to create a new updated master sample to use as a basis for sampling households. The selection of EAs was stratified by province and locality type. Locality types were urban formal, urban informal, rural formal (including commercial farms), and rural informal. In the formal urban areas, race type was also used as a third stratification variable (based on the predominant racial group in the selected EA at the time of the 2001 census).

The allocation of EAs to different stratification categories was disproportionate, that means, over-sampling or over-allocation of EAs occurred in areas that were dominated by Indian, Coloureds or White racial groups to ensure that the minimum required sample size in those smaller race groups is obtained. The selected 583 informed the primary sampling units (PSUs). Visiting points (VPs) or households were used as secondary sampling units (SSUs). Within each household, all eligible individuals (including consenting and non-consenting individuals) aged 18 to 24 years selected for the survey were the ultimate sampling unit (USU). To obtain an approximately self-weighted sample of visiting points (i.e. SSUs), the EAs were sampled with probability proportional to the size of the EA using the 2001 census estimate of the number of visiting points in the EA database as a measure of size (MOS).

A random sample of 12 VPs was selected from each of the 583 using a systematic sampling approach. A visiting point was defined as a stand with an address that might have one or more than one household. A household was defined as a group of people living and eating together from the same pot. In each household all eligible household members were invited to participate and interviewed. The survey included persons of ages 18 to 24 years living in South African households of the four (out of nine) selected provinces, KwaZuluNatal, Mpumalanga, Eastern Cape and Gauteng Province, providing an urban-rural representation of South Africa.

Ethical approval for the study was obtained from the Human Sciences Research Council Research Ethics Committee. Participants signed informed consent forms.

\section{Measures}

The main outcome variable was adolescent pregnancy, for females (12-19 years) and for males had impregnated a girl when they were an adolescent (12-19 years). Using a social-ecological model the following variables were assessed: individual variables (contraceptive knowledge, self-esteem, sense of future, control over life, and partner risk reduction self-efficacy), social variables (social network resources, peer pressure, sexual attitudes) and structural variables (poverty, formal education, accessibility to condoms and relationship control).

Pregnancy history information referred to the number of pregnancies (including miscarried and terminated pregnancies or pregnancies where the baby had died after birth) a respondent has ever had or he has had with his partner; adolescent pregnancy was defined as below 20 years of age. Contraceptive knowledge was assessed with 9 items, e.g., "Have you heard about the Pill that women can take every day to avoid becoming pregnant?" 
Response options were "Yes", or "No". (Cronbach alpha for the contraceptive knowledge index was 0.70 in this sample).

Ever contraceptive or condom use was assessed with the items pill, IUD, injectables, emergency contraceptive, male condom and female condom use. A contraceptive or condom use index was formed by adding up the six items (Cronbach alpha 0.72).

Reproductive communication was assessed with 5 items, e.g., "Did your mother ever talk to you about reproduction (how babies are made)?" Response options were "Yes" or "No" (Cronbach alpha 0.69).

Self-esteem: 10-item Rosenberg self-esteem scale $^{18}$, with a score of 14 or less indicating lowself-esteem (Cronbach alpha 0.64).

"Sense of future" (6 items) such as "I have a plan for the future." Response options were "agree" or "disagree". No sense of future was classified as those who indicated to all 6 items not to have any sense of future (Cronbach alpha 0.63).

Partner risk reduction self-efficacy: 4 items such as "Would you be able to avoid sex any time you didn't want it?" Response options were, "No, Probably no, Probably yes, Yes" (Cronbach alpha 0.73).

Social network resources (2 items) such as "I have a strong network of family that support me". Response options ranged from $1=$ strongly agree to $4=$ strongly disagree. Low social network resources were coded 2-5, medium 6-7 and high 8 .

Sexually permissive attitudes were assessed with 10 items, e.g., "It is acceptable for somebody to have sex before they are 18 years." Response options were $1=$ agree or $2=$ disagree. Low sexual permissive attitudes were coded 0 , medium 1-2 and high 3-10. Cronbach alpha for this sexual attitude index was 0.61 in this sample.

Peer pressure ( 2 items) such as "I feel pressure from friends to do things I don't want to." (Response options ranged from 1="Very often" to $4=$ "Never"). Low peer pressure was coded 2, medium 3-5 and high 6-8.

Female role pressure ( 3 items) such as "If I want a husband I have to get pregnant." (Cronbach alpha 0.65). Women's vulnerability (11 items), e.g., "If the woman gets pregnant, the man seldom takes responsibility." Response options ranged from $1=$ strongly agree to $4=$ strongly disagree. (Cronbach alpha 0.66).

Poverty: 6 items on the availability or nonavailability of shelter, fuel or electricity, clean water, medicines or medical treatment, food and cash income in the past week. Response options ranged from 1="Not one day" to 4="Every day of the week" Poverty was defined as higher scores on nonavailability of essential items (Cronbach alpha 0.70).

Accessibility of condoms: 1 question, "How easy is it for you to get condoms if you needed or wanted them?" Response options ranged from $1=$ very easy to $4=$ very difficult.

Relationship control: 4 items (for those never in a relationship, they were asked imagine to be) such as "Your partner has more control than you do in important decisions that affect your relationship" Response options ranged from 1="Strongly disagree" to $4=$ "Strongly agree." Higher scores on lack of relationship power were defined as lack of relationship control (Cronbach alpha 0.81).

Risk behaviour: Various questions that included the number of lifetime sexual partners, having had two or more sexual partners in the past year, inconsistent (not always) condom use with the last non-regular sexual partner, early sexual debut (below 15 years), ever forced to have sex, concurrent sexual relationships, sex with someone who is much older, sexual intercourse frequency and length of last relationship. In addition, ever illicit drug use was included as risk behaviour.

Alcohol use: Alcohol Use Disorder Identification Test (AUDIT-C) questionnaire, ${ }^{19}$ a measure of consumption of alcohol (i.e., the frequency of drinking, the quantity consumed at a typical occasion), and the frequency of heavy episodic drinking (i.e., consumption of five standard drinks, 60 grams alcohol, or more on a single occasion). Using a cut-off score of 5 or more hazardous or harmful drinking was defined. ${ }^{20}$ (Cronbach alpha 0.91).

\section{Data analysis}

The data were entered using Census Survey Processing software (CSPro). Data from the questionnaires were entered manually and verified. The verification process included double data entry of all questionnaires and its fields, doing programmed range checks by computer to identify outlying values, checking for missing values, and checking for inconsistencies in the data. Due to the sampling design of the survey some individuals have a greater or lesser probability of selection than others. To correct this problem, sample weights were introduced to correct for potential bias at the EA, household and individual levels and also adjust for non-response. Weighting procedures were undertaken before analysis of the data as follows: 
the data file of drawn EAs and sites contained the selection probabilities as well as the sampling weights of these EAs and sites. These weights reflect the disproportionate allocation of EAs and sites according to the stratification variables - race, locality type and province. The VP sampling weight was then calculated. This weight was computed as the counted number of VPs in the EA/site, proportionally corrected for invalid VPs and divided by the number of VPs participating in the survey. The final VP sampling weight was the product of the EA/site sampling weight and the VP sampling weight since all eligible persons in the household were invited to participate.

Weighted data were analysed using STATA software version 10. Data analysis consisted of both descriptive and inferential statistics. Estimates of values of key indicators, significance values ( $\mathrm{p}$-values) and confidence intervals (95\% CI) that take into account the complex design and individual sample weights were obtained. Computed estimates and odds ratios are reported with $95 \%$ confidence intervals and a two-side p-value of 0.05 used as the cut-off point for statistical significance. Associations between key outcome adolescent pregnancy and individual, social and structural variables and programme exposure were evaluated calculating odds ratios (OR). Unconditional multivariable logistic regression was used for evaluation of the impact of explanatory variables for key outcome of adolescent pregnancy (binary dependent variables). All variables statistically significant at the $\mathrm{p}<.05$ level in bivariate analyses were included in the multivariable models.

\section{Results}

\section{Survey response rate}

A total of 5768 households was sampled and approached for the interview. Among these 94.8\% households were valid. Among the valid households $93.6 \%$ agreed to be interviewed. Only households that indicated they had a person aged 18 to 24 years were eligible for an individual questionnaire administration. Of the eligible and valid households $47.2 \%$ were eligible for an individual interview, 1.3\% refused the individual interview and $2.3 \%$ individuals were absent from the household. Individual interview response rate was $96.4 \%$.

\section{Sample characteristics}

The total sample included 3123 participants, aged 18-24, 54.6\% men and 45.4\% women. Almost all participants were Black Africans (97.5\%) and about half $(51.7 \%)$ were Zulu speakers. The mean age of the sample was 20.5 years (SD=2.0). Overall, 61.4\% of young people reported having graduated from high school.

Among female youth $19.2 \%$ had had an adolescent pregnancy, and among male youth 5.8\% had impregnated a girl when they were an adolescent (12-19 years), $16.2 \%$ of the women ever had an unwanted pregnancy and $6.7 \%$ had ever terminated a pregnancy. Overall, 2.6\% $(n=96)$ reported to have been diagnosed with an STI in the past 12 months, and among those who had been tested for HIV and indicated their test result 7.4\% $(\mathrm{n}=109)$ were HIV positive; $106(7.3 \%)$ did not want to indicate their HIV status and $450(22.6 \%)$ had not been tested for HIV. Most young people had medium or high social network resources $(88.3 \%$ ) and a minority felt high pressure from their peers $(10.3 \%)$.

In general there seemed to be low poverty, $61.6 \%$ had secondary education or higher, $36.4 \%$ were unemployed, the degree of difficulty to access condoms was low and there was a moderate lack of relationship control. Almost one in five (19.1\%) engaged in hazardous or harmful alcohol use and $5.4 \%$ had ever used illicit drugs (table 1).

\section{Reasons for adolescent pregnancy}

Nineteen percent of respondents got pregnant because they wanted to prove their maturity or identity as women. However, most of the respondents $(55.5 \%)$ got pregnant the first time because they did not understand the risks involved in what they were doing or did not understand how pregnancy happens. Most respondents (74.1\%) indicated that their pregnancies were unwanted, yet only $6.8 \%$ ever terminated a pregnancy or persuaded someone to do so.

\section{Gender related perceptions}

Young women were asked about gender related perceptions, which may entrech unequal power relations between men and women. Some of the unequal power relations were confirmed by a sizable proportion of the young women. Over half of young women in this study $(55.9 \%)$ endorsed that they have to endure much greater hardship than men. A majority of $70.8 \%$ young women respondents agreed that women have more responsibilities than men, while more than one in five believed that they have fewer opportunities than boys after leaving school. One in three felt that they are unequal to males and four in five believed women were always vulnerable to violence and abuse. 
Table 1: Individual, social and structural variables and adolescent pregnancy of study sample by gender

\begin{tabular}{|c|c|c|}
\hline Individual & $\begin{array}{l}\text { Male } \\
\mathrm{N}(\%)\end{array}$ & $\begin{array}{l}\text { Female } \\
\mathrm{N}(\%)\end{array}$ \\
\hline Contraceptive knowledge (range 0-7) & $4.4(1.8)$ & $5.0(1.5)$ \\
\hline Ever made someone pregnant/pregnant & $243(22.1)$ & $479(42.7)$ \\
\hline Adolescent pregnancy (12-19 years) & $99(5.8)$ & $282(19.3)$ \\
\hline Unwanted pregnancy & $63(34.6)$ & $88(16.2)$ \\
\hline Persuaded someone to terminate pregnancy/terminated pregnancy & $29(6.8)$ & $38(6.7)$ \\
\hline Low self-esteem & $91(5.2)$ & $71(3.6)$ \\
\hline No sense of future & $495(24.9)$ & $432(32.8)$ \\
\hline Partner risk reduction self-efficacy (range 4-16) & $14.0(2.4)$ & $14.2(2.5)$ \\
\hline \multicolumn{3}{|l|}{ Social } \\
\hline \multicolumn{3}{|l|}{ Social network resources } \\
\hline Low & $156(8.1)$ & $164(15.9)$ \\
\hline Medium & $840(55.5)$ & $707(53.4)$ \\
\hline High & $527(36.4)$ & $458(30.8)$ \\
\hline \multicolumn{3}{|l|}{ Peerpressure } \\
\hline Low & $558(43.0)$ & $679(56.7)$ \\
\hline Medium & $744(44.4)$ & $523(35.8)$ \\
\hline High & 247 (12.7) & $153(7.5)$ \\
\hline \multicolumn{3}{|l|}{ Sexual attitudes } \\
\hline Low & $357(22.5)$ & $423(33.1)$ \\
\hline Medium & $586(40.0)$ & $568(42.5)$ \\
\hline High & $600(37.4)$ & $354(24.4)$ \\
\hline Talked with partner about condoms in past 12 months & $903(91.9)$ & $769(90.7)$ \\
\hline Female role pressure (range 3-12) & $\mathrm{n} / \mathrm{a}$ & $3.8(1.7)$ \\
\hline Female reproduction communication (range $0-5$ ) & $\mathrm{n} / \mathrm{a}$ & $3.5(1.4)$ \\
\hline \multicolumn{3}{|l|}{ Structural } \\
\hline Poverty index (range 6-24) & $8.3(3.0)$ & $7.9(2.8)$ \\
\hline \multicolumn{3}{|l|}{ Education } \\
\hline$\leq$ Grade 11 & $702(37.9)$ & $552(37.8)$ \\
\hline Grade 12 or more & $890(62.1)$ & $835(61.6)$ \\
\hline Student & $772(50.3)$ & $614(51.3)$ \\
\hline Employed & $237(16.1)$ & $134(9.0)$ \\
\hline Unemployed & $471(33.5)$ & $521(39.8)$ \\
\hline Difficulty of getting condoms (range 1-4) & $1.22(0.6)$ & $1.18(0.6)$ \\
\hline Women vulnerability index (range 11-44) & $\mathrm{n} / \mathrm{a}$ & $26.5(4.7)$ \\
\hline Lack of relationship control (range 4-16) & $8.4(2.4)$ & $8.0(2.5)$ \\
\hline \multicolumn{3}{|l|}{ Risk status and behaviour } \\
\hline Diagnosed HIV positive & $40(5.8)$ & $69(8.5)$ \\
\hline Lifetime diagnosed with an STI & $128(5.3)$ & $113(7.1)$ \\
\hline Early sex $(<15)$ & $145(17.8)$ & $65(6.9)$ \\
\hline Ever forced sex & $14(1.1)$ & $58(7.3)$ \\
\hline Number of lifetime sexual partners (range 0-75 for men and 0-30 for women) & $3.3(5.9)$ & $1.7(2.6)$ \\
\hline Sex with much older & $354(21.5)$ & $326(17.7)$ \\
\hline Hazardous or harmful alcohol use & $453(24.3)$ & $173(12.9)$ \\
\hline Ever drugs & $171(8.3)$ & $34(2.0)$ \\
\hline \multicolumn{3}{|l|}{ Contraceptive or condom use } \\
\hline Ever contraceptive pill & $261(15.2)$ & $249(14.7)$ \\
\hline Ever IUD & $16(0.4)$ & $19(1.5)$ \\
\hline Ever injectables & $348(18.2)$ & $508(33.2)$ \\
\hline Ever male condoms & $1004(61.5)$ & $842(59.4)$ \\
\hline Ever female condoms & $96(4.1)$ & $79(45.0)$ \\
\hline Ever rhythm method & $134(6.7)$ & $127(8.3)$ \\
\hline Ever withdrawal & $333(22.4)$ & $265(17.0)$ \\
\hline Ever emergency contraception & $170(11.8)$ & $188(10.4)$ \\
\hline Ever dual method (contraception and condom) & $139(10.5)$ & $190(12.8)$ \\
\hline
\end{tabular}


Sexual and reproductive health information and services for young women

The young women in this study were asked about communication on various sources about reproduction (how babies are made) and use of reproductive health services. Eighty-five percent of young women reported that their teacher had talked to them about reproduction, followed by the clinic nurse (74.1\%) and family members (mother: 68.5\%, sister: $67.9 \%$ and auntie or older woman: $58.3 \%$ ). The majority of the young women $(58.5 \%)$ had ever attended a clinic for reproductive health services (e.g. contraception, HIV testing).

Table 2: Association between individual, social and structural variables, risk status and behaviour and adolescent pregnancy of young women

\begin{tabular}{|c|c|c|}
\hline \multirow[b]{2}{*}{ Individual } & \multicolumn{2}{|c|}{ Adolescent pregnancy-female } \\
\hline & $\mathrm{OR}(95 \% \mathrm{CI})$ & AOR $(95 \% \mathrm{CI})$ \\
\hline Contraceptive knowledge & $0.77(0.56-1.05)$ & - \\
\hline Unwanted pregnancy & $1.89(0.77-4.12)$ & - \\
\hline Low self-esteem & $0.98(0.48-2.01)$ & -- \\
\hline No sense of future & $0.78(0.30-2.01)$ & - \\
\hline Partner risk reduction self-efficacy & $0.98(0.88-1.10)$ & - \\
\hline \multicolumn{3}{|l|}{ Social } \\
\hline \multicolumn{3}{|l|}{ Social network resources } \\
\hline Low & 1.00 & 1.00 \\
\hline Medium & $0.32(0.18-0.56)^{* * *}$ & $0.59(0.24-1.43)$ \\
\hline High & $0.31(0.13-0.75)^{* *}$ & $0.54(0.24-1.22)$ \\
\hline \multicolumn{3}{|l|}{ Peerpressure } \\
\hline Low & 1.00 & \\
\hline Medium & $1.25(0.73-2.11)$ & \\
\hline High & $0.93(0.42-2.02)$ & - \\
\hline \multicolumn{3}{|l|}{ Sexually permissive attitudes } \\
\hline Low & 1.00 & 1.00 \\
\hline Medium & $1.59(0.84-3.01)$ & $1.81(0.98-3.37)$ \\
\hline High & $5.20(1.86-14.53)^{* *}$ & $3.33(1.24-8.99)^{*}$ \\
\hline Female role pressure & $0.87(0.35-2.19)$ & - \\
\hline Female reproduction communication & $0.85(0.59-1.22)$ & -- \\
\hline \multicolumn{3}{|l|}{ Structural } \\
\hline Poverty index & $1.14(1.02-1.29)^{*}$ & $1.14(1.02-1.26)^{*}$ \\
\hline Education & $0.96(0.27-3.43)$ & 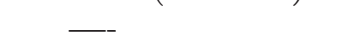 \\
\hline Student & 1.00 & 1.00 \\
\hline Employed & $5.19(2.31-11.65)^{* * *}$ & $5.74(2.31-15.48)^{* *}$ \\
\hline Unemployed & $5.72(2.32-14.04)^{* * *}$ & $4.05(1.77-9.26)^{* * *}$ \\
\hline Difficulty of getting condoms & $0.74(0.47-1.18)$ & - \\
\hline Women vulnerability index & $1.03(0.98-1.09)$ & - \\
\hline Lack of relationship control & $1.05(0.91-1.21)$ & - \\
\hline \multicolumn{3}{|l|}{ Risk status and behaviour } \\
\hline HIV positive (vs. negative) & $1.50(0.45-4.98)$ & - \\
\hline Lifetime STI & $2.16(0.80-5.82)$ & - \\
\hline Early sex $(<15)$ & $1.68(0.46-6.09)$ & 一 \\
\hline Ever forced sex & $1.08(0.47-2.44)$ & -- \\
\hline Number of lifetime sexual partners & $1.00(0.98-1.02)$ & - \\
\hline Hazardous or harmful alcohol use & $5.00(1.46-17.07)^{* *}$ & $1.17(0.45-3.05)$ \\
\hline Ever drugs & $0.43(0.10-1.81)$ & - \\
\hline Contraceptive or condom use index & $1.42(1.11-1.83)^{* *}$ & $1.45(1.07-1.99)^{*}$ \\
\hline
\end{tabular}

\section{Predictors of adolescent pregnancy}

In multivariable analysis among women it was found that being employed or unemployed, greater poverty, having higher sexually permissive attitudes and scoring higher on the contraceptive or condom use index was associated with adolescent pregnancy. In multivariable analysis among men wanting the pregnancy and having a sense of future were associated with adolescent pregnancy (see tables 2 and 3). 
Table 3: Association between individual, social and structural variables, risk status and behaviour and adolescent pregnancy of male partner

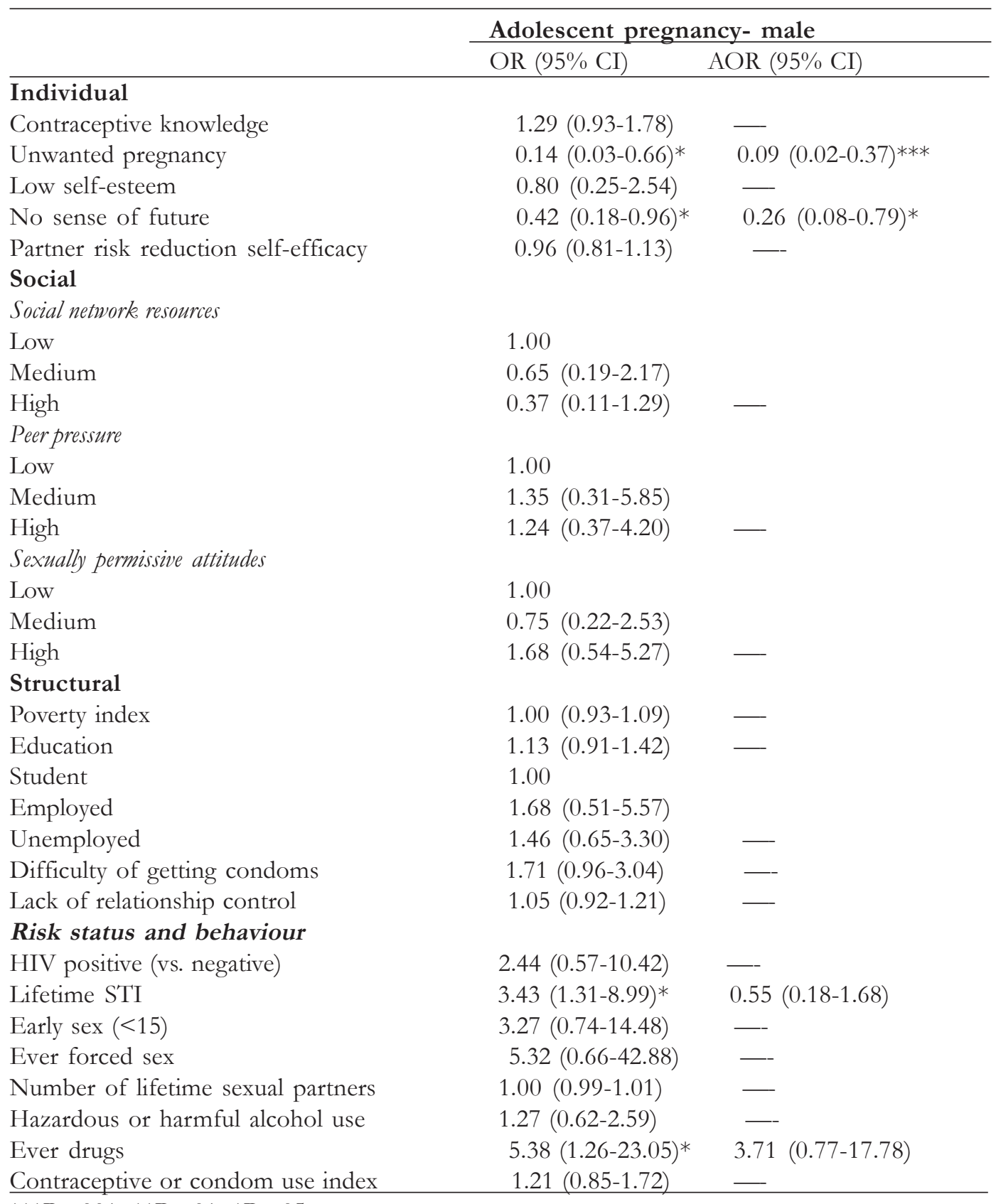

$* * * \mathrm{P}<.001 ; * * \mathrm{P}<.01 ; * \mathrm{P}<.05$

\section{Discussion}

While previous research findings have suggested that individual factors and other predictors such as poverty, low educational level, contributed to young women engaging in risky sexual behaviour, leading to unplanned pregnancy in adolescents, $2,3,7,8,21$ the findings of this study, showed that more than half of female respondents $(74.1 \%)$ fell pregnant due to lack of knowledge, and $55 \%$ of respondents got pregnant because they did not understand the risks involved. However, what is equally a social concern is the fact that $71.2 \%$ of the female respondents did not understand how pregnancy happens or did not think about risks involved in engaging in unprotected sexual intercourse (which could explain the high number of unwanted pregnancies). This finding is a concern because it shows that young South African youth still engage in risky sexual behaviours, not only due to lack of knowledge but due to unfavourable 
decisions. It is worth noting that these respondents are between the ages of 18-24 years, thus it is highly unexpected that the majority of them do not understand the risks involved or how pregnancy happens particularly when taking into account number of various intervention programmes including Life Orientation (which was introduced in 2000) that have been implemented.

Similar to the findings of the Department of Health study, ${ }^{22}$ the findings of the current study did not confirm child-support grant as a possible enticement for girls to become pregnant. ${ }^{5,6}$ Our findings however did indicate that lack of employment and job opportunities was associated with teenage pregnancy. Although the current study still shows that a low percentage of women are utilizing condoms, it was encouraging that young women are becoming more assertive in negotiating for condom usage as demonstrated in some previous studies. ${ }^{23,24}$ This finding was contrary to the findings of other previous studies that young women have little or no negotiating power with their partners to insist on condoms usage in relationships. ${ }^{25,26}$ However, this finding might be different in a transactional sexual relationship since quantitative $e^{10-13}$ and qualitative research findings have shown that women often assert that accepting financial or material assistance from a man means accepting sex on his terms, which very often means sex without condoms. ${ }^{27}$

Although the findings show that $69 \%$ of female respondents felt that they would be able to use a condom every time they had sexual intercourse, the findings still showed that only $9.5 \%$, compared to $36.9 \%$ of their male counterparts used a condom when they were with their non- regular partner, a behaviour which puts them more at risk. This finding could be an indication that even though women feel empowered to negotiate condom use, it might not be as easy when they are with their partners. This finding may be an indication for another related study exploring the reasons for this failure to negotiate condom use, while women feel they are empowered enough to do so. An interesting finding was that the majority young women did not feel pressured or coerced to engage in sexual activities $(86.8 \%)$, to get married $(78.7 \%)$ and even to fall pregnant $(87.4 \%)$. This finding was contrary to the findings of previous studies that many young women have unprotected heterosexual intercourse because they feel pressured to maintain a "good" female (sexual) reputation amongst peers. ${ }^{3,28}$

\section{Conclusion}

Adolescent pregnancy was found to be high in this sample of South African youth. Multiple factors contributing to adolescent pregnancy have been identified which can be used in targeting young people on the prevention of adolescent pregnancy.

\section{Acknowledgements}

This research was a collaborative project between loveLife and the HSRC, and was made possible by the Henry J. Kaiser Family Foundation. We would like to acknowledge loveLife and the anonymous review committee for their comments and suggestions. We would also like to thank the research participants who took part in the study.

\section{References}

1. World Health Organisation (WHO). Adolescent pregnancy, 2011. Retrieved from http:// www.who.int/making_pregnancy_safer/ topics/adolescent_pregnancy/en/index.html at 14 December 2011.

2. Omar K, Hasim S, Muhammad NA, Jaffar A, Hashim SM, Siraj HH. Adolescent pregnancy outcomes and risk factors in Malaysia. Int $\mathrm{J}$ Gynaecol Obstet 2010;111:220-223.

3. East PL, Khoo ST, Reyes BT. Risk and protective factors predictive of adolescent pregnancy: A longitudinal, prospective study. Appl Dev Sci 2006;10(4):188-199.

4. Mkhwanazi N. Teenage pregnancy and HIV in South Africa. In Schlyter A (Ed) Body politics and women citizens: African experiences (pp.83-92). Retrieved from http://www.sida.se/ Documents/Import/pdf/Sida-Studies-No-24Body-Politics-and-Women-Citizens.pdf at 14 December 2011.

5. Bankole A, Malarcher S. Removing barriers to adolescents' access to contraceptive information and services. Stud Fam Plann 2010;41(2):117-124.

6. Buchmann EJ, Mensah K, Pillay P. Legal termination of pregnancy among teenagers and older women in Soweto, 1999-2001. S Afr Med J 2002;92:729-731.

7. Klein JD, American Academy of Pediatrics Committee on Adolescence. Adolescent pregnancy: Current trends and issues. Pediatrics 2005;116(1):281-286.

8. Pedrosa AA, Pires R, Carvalho P, Canavarro MC, Dattilio F. Ecological contexts in adolescent 
pregnancy: The role of individual, sociodemographic, familial and relational variables in understanding risk of occurrence and adjustment patterns. Contemp Fam Ther 2011;33:107-127.

9. Grant MJ, Hallman KK. Pregnancy-related school dropout and prior school performance in KwaZulu-Natal, South Africa. Stud Fam Plann 2008;39(4):369-382.

10. Bledsoe C. School fees and the marriage process for Mende girls in Sierra Leone. In Sanday PR, Goodenough RG (Eds). Beyond the second sex: Essays in the anthropology of gender (pp. 283-305). Philadelphia, Pennsylvania: University of Pennsylvania Press, 1990.

11. Castle S, Konaté MK. The context and consequences of economic transactions associated with sexual relations among Malian adolescents. The African Population in the 21st Century: Proceedings of the Third African Population Conference 2: 105-128. Durban, 1999. Retrieved from http:// www.matrix.msu.edu/ ucad/papadembafall/ maoumy/Cap\%2099.htm, at 20 March 2012.

12. Krishnan S, Dunbar MS, Minnis AM, Medlin CA, Gerdts CE, Padian NS. Poverty, gender inequities, and women's risk of Human Immunodeficiency Virus/AIDS. Ann NY Acad Sci 2008;1136(1):101-110.

13. Luke N. Confronting the 'Sugar Daddy' stereotype: age and economic asymmetries and risky sexual behavior in urban Kenya. Int Fam Plann Perspect 2005;31:6-25.

14. Chatterji M, Murray N, London D, Anglewicz P. The factors influencing transactional sex among young men and women in 12 sub-Saharan African countries. Soc Biol 2005; 52(1-2):56-72.

15. Gregson S, Nyamukapa C A, Garnett G P, et al. Sexual mixing patterns and sex-differentials in teenage exposure to HIV infection in rural Zimbabwe. Lancet 2002;359:1896-903.

16. Longfield K, Glick A, Waithaka M, Berman J. Relationships between older men and younger women: implications for STIs/HIV in Kenya. Stud Fam Plann 2004;35(2):125-34.

17. Regmi K. Opportunities and challenges of sexual health services among young people: A study in Nepal. J Sex Med 2009;6:352-361.

18. Vundule C, Maforah N F, Jewkes R, Jordaan E. Risk factors for teenage pregnancy amongst African adolescents in metropolitan Cape Town: A case control study. S Med J 2001;91:73-80.
19. Rosenberg M. Society and the adolescent self-image. Princeton, NJ: Princeton University Press, 1965.

20. Freeborn DK, Polen MR, Hollis JF, Senft RA. Screening and brief intervention for hazardous drinking in an HMO: effects on medical care utilization. J Behav Health Serv Res 2000;27(4):44653.

21. Gual A, Segura L, Contel M, Heather N, Colom J. AUDIT-3 and AUDIT-4: Effectiveness of two short forms of the alcohol use disorders identification test. Alcohol Alcohol 2002;37(6): 561-596.

22. Pettifor AE, Levandowski BA, MacPhail C, Padian NS, Cohen MS, Rees HV. Keep them in school: the importance of education as a protective factor against HIV infection among young South African women. Int J Epidemiol 2008;37(6):1266-1273.

23. MacPhail C, Pettifor AE, Pascoe S, Rees HV. Contraception use and pregnancy among 1524 year old South African women: a nationally representative cross-sectional survey. BMC Medicine 2007;5:31.

24. MacPhail C, Campbell C. 'I think condoms are good but, aai, I hate those things': condom use among adolescents and young people in a southern African township. Soc Sci Med 2001;52(11):1613-1627.

25. Wingood GM, DiClemente RJ. The effects of an abusive primary partner on the condom use and sexual negotiation practices of AfricanAmerican women. Am J Public Health 1997;87(6):1016-1018.

26. Leclerc-Madlala S. Age-disparate and intergenerational sex in southern Africa: the dynamic of hypervulnerability. AIDS 2008;22(Suppl. 4):17-25.

27. Gregson S, Nyamukapa CA, Garnett, GP et al. Sexual mixing patterns and sex-differentials in teenage exposure to HIV infection in rural Zimbabwe. Lancet 2002;359:1896-1903.

28. Dunkle KL, Jewkes RK, Brown HC, et al. Transactional sex among women in Soweto, South Africa: prevalence, risk factors and association with HIV infection. Soc Sci Med 2004;59:1581-1592.

29. Kumar N, Larkin J, Mitchell C. Gender, youth and HIV risk. Canadian Woman Stud J 2001;21(2):35-43. 